\title{
CORE: A randomised trial of COnventional care versus Radioablation (stereotactic body radiotherapy) for Extracranial oligometastases
}

\author{
V. Khoo ${ }^{1}$, M. Ahmed ${ }^{1}$, F. McDonald ${ }^{1}$, A. Kirby ${ }^{1}$, N. Van As ${ }^{1}$, M. Hawkins ${ }^{2}$, I. Syndikis ${ }^{3}$, K. \\ Franks ${ }^{4}$, S. Jain ${ }^{5}$, A. Tree ${ }^{1}$, D. Eaton ${ }^{6}$, R. Patel6, M. Yip Braidley ${ }^{7}$, L. Kilburn ${ }^{7}$, C. Toms ${ }^{7}$, E. Hall ${ }^{7}$ \\ ${ }^{1}$ Clinical Oncology, The Royal Marsden NHS Foundation Trust, London, United \\ Kingdom; ${ }^{2}$ Clinical Oncology, Churchill Hospital, Oxford, United Kingdom; ${ }^{3}$ Clinical Oncology, \\ Clatterbridge Centre for Oncology, Liverpool, United Kingdom; ${ }^{4}$ Leeds Cancer Centre, St \\ James's University Hospital, Leeds, United Kingdom; ${ }^{5}$ Clinical Oncology, Northern Ireland \\ Cancer Centre, Belfast, United Kingdom; ${ }^{6}$ RTTQA, Mount Vernon Hospital, London, United \\ Kingdom; ${ }^{7}$ CTSU, The Institute of Cancer Research, London, United Kingdom.
}

Introduction: Retrospective and prospective cohort studies indicate SBRT treatment of oligometastatic disease is associated with higher rates of local control with acceptably toxicity. A lack of randomised data limits interpretation preventing evaluation of

the true benefit of adding SBRT to systemic therapy. The potential therapeutic benefit of adding SBRT to standard therapy may vary between tumour sites and there is insufficient evidence currently to predict which cancers may benefit most from such an approach.

CORE will investigate whether the addition of SBRT to standard therapy improves survival outcomes, focusing on common primary tumour sites where oligometastatic disease is encountered (Breast, prostate and NSCLC). CORE will aim to demonstrate: Feasibility of recruitment; Deliverability of the study in a multi-centre setting; Activity of SBRT based on progression free survival across three tumour types.

Methods: CORE is a CRUK funded, phase II/III multi-centre, nonblinded, randomised controlled trial in patients with breast, prostate or NSCLC primary cancer comparing standard of care (SOC) with or without SBRT for extra-cranial metastases. Eligible patients will have either primary breast, prostate or NSCLC presenting with $\leq 3$ extra-cranial, metachronous, oligometastases all suitable for SBRT. Patients randomised 1:1 to either SOC or SOC + SBRT. Choice of SOC at local clinician's discretion, defined prior to randomisation.

Conclusion: Since the introduction of IMRT, there has been a steady increase in the proportion of patients treated with curative-intent across all PS groups and stages of disease. This may be attributed to IMRT, as it enables us to treat large volumes and tumours in proximity to organs at risk that historically would have been treated palliatively. However other factors such as stereotactic radiotherapy, satellite radiotherapy centres, improved staging and a change in staging classification may also have contributed. Work is on-going to examine the impact of IMRT on survival in this heterogeneous patient population.

Disclosure: All authors have declared no conflicts of interest. 